\title{
USO DEL CONOCIMIENTO CIENTÍFICO EN ACADÉMICOS DE UNA INSTITUCIÓN DE EDUCACIÓN SUPERIOR
}

\author{
Carolina VARGAS-ALMONACID ${ }^{1}$, \\ Rubén VIDAL-ESPINOZA ${ }^{2}$ \\ Lorena JIMÉNEZ-OLIVA ${ }^{3}$ \\ Rossana GÓMEZ-CAMPOS ${ }^{4}$ \\ Marco COSSIO-BOLAÑOS ${ }^{5}$
}

\begin{abstract}
Resumen
La formación científica en las instituciones de educación superior, es fundamental para impulsar la investigación científica entre sus estudiantes. El objetivo del presente trabajo fue verificar las diferencias del uso del conocimiento científico por género, grado académico y experiencia docentes y buscar sus posibles relacione entre variables.
\end{abstract}

Fecha de recepción del artículo: Abril 2020

Fecha de evaluación: Mayo 2020

\footnotetext{
${ }^{1}$ Magister en psicopedagogía, Universidad Tecnológica de Chile INACAP. Email: cvargas@hotmail.com

2 Profesor de Educación Especial y Diferenciada y Magister en Educación Especial de la Pontificia Universidad Católica de Chile. Académico e Investigador de la Carrera de Educación Diferencial de la Universidad Católica Silva Henríquez, Santiago -Chile. Email: rvidale@ucsh.cl.

${ }^{3}$ Coordinadora de Especialidad de la Universidad Tecnológica de Chile INACAP. Email: geisade87@gmail.com

${ }^{4}$ Doctora en Biodinámica del movimiento por la Universidad Estadual de Campinas, Brasil. Docente investigador del departamento de Diversidad e Inclusividad educativa de la Universidad Católica de Maule Av. San Miguel s/n, Talca, Chile. Email: rossaunicamp@gmail.com

${ }^{5}$ Universidad Católica del Maule. Talca. Chile Profesor de Educación Física. Universidad Nacional de San Marco. Perú. Doctor en Ciencias del Deporte en la Universidad Estadual de Campinas. Brasil. Universidad Nacional de San Marco. Perú. Doctor en Ciencias del Deporte. Universidad Estadual de Campinas. Brasil. Es autor de un número significativo de artículos en revistas indexadas de alto impacto.

mcossio1972@hotmail.com
} 
Se efectuó un estudio descriptivo correlacional en 75 académicos (45 mujeres y 30 hombres). Se aplicó un cuestionario que mide el uso del conocimiento científico UCC (11 preguntas). El estudio demostró que la experiencia profesional es determinante en el UCC en una universidad regional, por lo que los resultados sugieren que las instituciones de educación superior deben vincular investigadores para fortalecer la alfabetización científica de los alumnos del pregrado.

Palabras claves: Conocimiento científico, Universidad, académicos.

\begin{abstract}
Scientific training in higher education institutions is essential to promote scientific research among its students. The objective of the present work was to verify the differences in the use of scientific knowledge by gender, academic degree and teaching experience and to look for its possible relationship between variables. A descriptive correlational study was carried out on 75 academics ( 45 women and 30 men). A questionnaire measuring the use of UCC scientific knowledge was applied (11 questions). The study showed that professional experience is decisive at the UCC in a regional university, so the results suggest that higher education institutions should link researchers to strengthen the scientific literacy of undergraduate students.
\end{abstract}

Key words: Scientific knowledge, University, academics.

\title{
Résumé
}

La formation scientifique dans les établissements d'enseignement supérieur est essentielle pour promouvoir la recherche scientifique auprès de ses étudiants. L'objectif du présent travail était de vérifier les différences dans l'utilisation des connaissances scientifiques par sexe, diplôme universitaire et expérience d'enseignement et de rechercher sa relation possible entre les variables. Une étude corrélationnelle descriptive a été menée auprès de 75 universitaires (45 femmes et 30 hommes). Un questionnaire mesurant l'utilisation des connaissances scientifiques de l'UCC a été appliqué (11 questions). L'étude a montré que l'expérience professionnelle est décisive à l'UCC dans une université régionale, de sorte que les résultats suggèrent que les établissements d'enseignement supérieur devraient relier les chercheurs pour renforcer la culture scientifique des étudiants de premier cycle.

Mots clés: connaissances scientifiques, université, universitaires.

\section{INTRODUCCIÓN}

La formación científica en las instituciones de educación superior, es uno de los requisitos fundamentales para impulsar la investigación científica entre sus estudiantes. De hecho, los educadores e investigadores deben participar en el proceso de la alfabetización a los estudiantes para sensibilizar e impulsar la investigación. 
Tal es el caso, que la generación de nuevos conocimientos es responsabilidad de las universidades, en el que los docentes e investigadores en general deben enseñar, difundir y promover la investigación científica entre sus estudiantes (Cossio-Bolaños et al, 2014). Aunque actualmente las universidades enfrentan desafíos para garantizar la alfabetización científica por falta de profesionales calificados, surge la necesidad de contar con profesionales vinculados a la investigación.

De hecho, estos profesionales deben lograr la alfabetización científica, a través de la cual, permitan a los estudiantes reconocer, no sólo los principales conceptos, hipótesis y teorías de la ciencia, sino que sean capaces de usar en la resolución de problemas, la comprensión del mundo y la toma de decisiones (Sanudo \& Perales, 2014).

Los educadores en general, tienen el objetivo común de ayudar a los estudiantes a desarrollar la alfabetización científica, incluida la comprensión de la naturaleza de las ciencias (Miller et al., 2010). En ese sentido, el uso del conocimiento científico en la educación superior no sólo se refiere al dominio epistemológico, sino también implica el manejo y dominio de una serie de habilidades investigativas, por ejemplo, Ziatone (2013) resalta, la resolución de problemas, pensamiento crítico, adaptación al cambio en la ciencia y sus aplicaciones, aumentar la confianza de la comunidad en el valor del conocimiento y la importancia de la ciencia, la tecnología y su relación con la comunidad y la superposición con la comunidad.

En consecuencia, contar con herramientas, habilidades y sólidos conocimientos científicos en investigación, por un lado, puede ayudar a crear el valor del uso de la ciencia en los estudiantes universitarios, sin embargo, por otro lado, corre el riesgo de no tener respuesta, lo que probablemente puede desencadenar en el investigador en una necesidad de sobrevivir en la carrera académica (Beck et al., 2019) o de lo contrario en la deserción del mismo.

Actualmente las instituciones de educación superior son valoradas por la capacidad de generar conocimientos y nuevos saberes en diversas áreas del saber humano (CossioBolaños et al., 2014), lo que menudo está a cargo de investigadores que deben mantenerse actualizados, estar en pleno desarrollo de proyectos de investigación, redacción de artículos, participación en debates (Jamali \& Nicholas, 2008), entre otras actividades, y que por lo general están a cargo de investigadores senior.

Basados en estas premisas, esta investigación supone que el uso del conocimiento científico en académicos de una universidad de Chile, podrían estar relacionadas con la edad y los años de experiencia, dado que al parecer la edad y la experiencia juega un papel relevante.

Por lo tanto, el objetivo del estudio fue verificar las diferencias del uso del conocimiento científico por género, grado académico y experiencia docentes y buscar sus posibles relaciones entre variables.

\section{METODOLOGÍA}

Tipo de estudio y Muestra

Se efectuó un estudio descriptivo (comparativo-correlacional). La selección de la muestra fue de tipo no-probabilístico (por conveniencia). Participaron de este estudio 75 académicos 
que se encontraban con contrato vigente durante el período académico 2019. El rango de edad oscila entre 26 a 55 años.

Se invitó a participar del estudio de forma voluntaria, donde cada uno de los académicos firmó el consentimiento informado, además se efectuó de acuerdo a la declaración de Helsinki para seres humanos y conforme al comité de ética local. Se incluyeron a los que respondieron todas las preguntas. Se excluyeron a los académicos que tenían contrato con la Institución por menos dos años de antigüedad.

La institución cuenta con 7 escuelas: Escuela de Ingeniería y Tecnología, Escuela de Administración y Negocios, Escuela de Humanidades y Educación, Escuela Agropecuaria y Agroindustrial, Escuela de Mecánica y Electromovilidad, Escuela de Hotelería, Turismo y Gastronomía y por último Escuela de Salud. Las características de la muestra se encuentran descritas en la tabla 1.

\section{Técnica y Procedimientos}

Para este estudio se utilizó la técnica de la encuesta para medir la variable uso de conocimiento científico (UCC). El instrumento aplicado fue el cuestionario propuesto por Cossio, Vidal \& Valenzuela (2014). El instrumento cuenta con tres indicadores: Búsqueda de Información, Transferencia del Conocimiento y Contribución del Conocimiento, con un total de 11 preguntas, cuyas alternativas responden a una escala de frecuencia.

Se calculó la confiabilidad por consistencia interna, y el instrumento reflejó un alpha de r= 0,82 , lo que garantiza la fiabilidad del instrumento.

Se utilizó modalidad online para la recogida de datos, a través de formulario en línea, el que estuvo disponible por 30 días. Todos los académicos dispusieron de 15 minutos para responder las alternativas del cuestionario.

\section{Análisis estadístico}

Para analizar los datos del estudio se utilizó estadística descriptiva de frecuencia (fi), porcentajes (\%), promedio (x) y desviación estándar. Para la comparación entre grupos según variable sexo se utilizó la Prueba $t$ de Student para muestras independientes. Las diferencias entre grupos según variable grado académico y años de experiencia docente fueron verificadas por Anova de una vía y la prueba de especificad de Tukey. La relación entre variables se verificó por medio de Spearman. En todos los casos se adoptó p $<0.05$. Los cálculos se analizaron en planillas de Excel y SPSS 18.0.

\section{RESULTADOS}

En la tabla 1 se observa los indicadores que caracterizan la muestra estudiada. Los rangos de edad en ambos sexos oscilan desde los 25 hasta los 65 años y la mayor proporción de docentes se encuentran entre los 30 a 39 años. En cuanto al grado académico, la mayoría de los docentes se encuentras distribuidos entre licenciados (44\%) y pos-grado (45\%). En relación a la experiencia profesional, la mayoría se concentra entre 6 a 10 años de experiencia (37\%). 
Tabla 1. Características de la muestra estudiada

\begin{tabular}{lrcc}
\hline Indicadores & & $\mathbf{f i}$ & $\mathbf{\%}$ \\
\hline Sexo: & & \\
& Femenino & 30 & 40 \\
Masculino & 45 & 60 \\
Edades: & Total & 75 & 100 \\
& & & \\
& $<30$ & 7 & 9 \\
$30-39$ & 37 & 49 \\
$40-50$ & 20 & 27 \\
>50 & 11 & 15 \\
Total & 75 & 100 \\
Grado académico: & & \\
Sin Grado & 8 & 11 \\
Licenciatura & 33 & 44 \\
Pos grado & 34 & 45 \\
Total & 75 & 100 \\
Experiencia docente & & \\
1 a 5 años & 25 & 33 \\
6 a 10 años & 28 & 37 \\
11 a 15 años & 8 & 11 \\
Más de 15 años & 14 & 19 \\
Total & 75 & 100 \\
\hline
\end{tabular}

Leyenda: Fi: Frecuencia, \%: Porcentaje.

Las comparaciones del UCC se observan en la tabla 2. No hubo diferencias significativas entre ambos sexos, entre grados académicos y entre años de experiencia profesional en las tres dimensiones del instrumento aplicado ( $\mathrm{p}>0.05)$. 
Tabla 2. Comparación del uso del conocimiento científico (UCC) por sexo, grado académico y experiencia docente.

\begin{tabular}{|c|c|c|c|c|c|c|c|c|}
\hline \multirow{2}{*}{ Indicadores } & \multicolumn{2}{|c|}{$\begin{array}{l}\text { Búsqueda } \\
\text { Información }\end{array}$} & \multicolumn{2}{|c|}{$\begin{array}{l}\text { Transferencia } \\
\text { Conocimiento }\end{array}$} & \multicolumn{2}{|c|}{$\begin{array}{l}\text { Contribución } \\
\text { conocimiento }\end{array}$} & \multicolumn{2}{|c|}{ Total, UCC } \\
\hline & $\mathbf{X}$ & DE & $\mathbf{X}$ & DE & $\mathbf{X}$ & DE & $\mathbf{X}$ & DE \\
\hline \multicolumn{9}{|l|}{ Sexo: } \\
\hline Femenino & 5,8 & 0,8 & 10,9 & 1,2 & 9,8 & 1,9 & 25,7 & 2,9 \\
\hline Masculino & 5,1 & 0,8 & 11,1 & 1,8 & 10,1 & 1,6 & 26,3 & 3,4 \\
\hline Ambos & 5,0 & 0,8 & 11,0 & 1,6 & 10,0 & 1,7 & 26,1 & 3,2 \\
\hline \multicolumn{9}{|l|}{ Grado académico } \\
\hline Sin grado & 5,1 & 0,6 & 9,9 & 2,2 & 9,4 & 1,7 & 24,4 & 4,0 \\
\hline Licenciado & 5,1 & 0,8 & 11,1 & 1,6 & 10,2 & 1,7 & 26,4 & 3,3 \\
\hline Post Grado & 5,0 & 0,9 & 11,3 & 1,5 & 9,9 & 1,8 & 26,2 & 3,0 \\
\hline \multicolumn{9}{|c|}{ Experiencia Docente } \\
\hline 1 a 5 años & 5,2 & 0,8 & 11,2 & 1,3 & 10,2 & 1,5 & 26,7 & 3,6 \\
\hline 6 a 10 años & 5,4 & 2,0 & 11,0 & 1,4 & 11,1 & 4,8 & 27,5 & 8,3 \\
\hline 11 a 15 años & 5,5 & 0,8 & 11,0 & 2,6 & 10,1 & 2,2 & 26,6 & 5,5 \\
\hline Más de 16 años & 4,7 & 1,1 & 10,7 & 1,9 & 9,6 & 1,9 & 25,0 & 4,8 \\
\hline
\end{tabular}

Leyenda: X: Promedio, DE: Desviación estándar, UCC: Uso del conocimiento científico, Pos grado: Magister + Doctorado, NS: No significativo

La relación entre la edad y años de experiencia docente con el UCC (total) se observan en la figura 1. Hubo correlación positiva entre la edad con el $\mathrm{UCC}(\mathrm{r}=0.12, \mathrm{p}<0.05)$ y entre los años de experiencia docente con el UCC $(r=-0.80, \mathrm{p}<0.05)$.
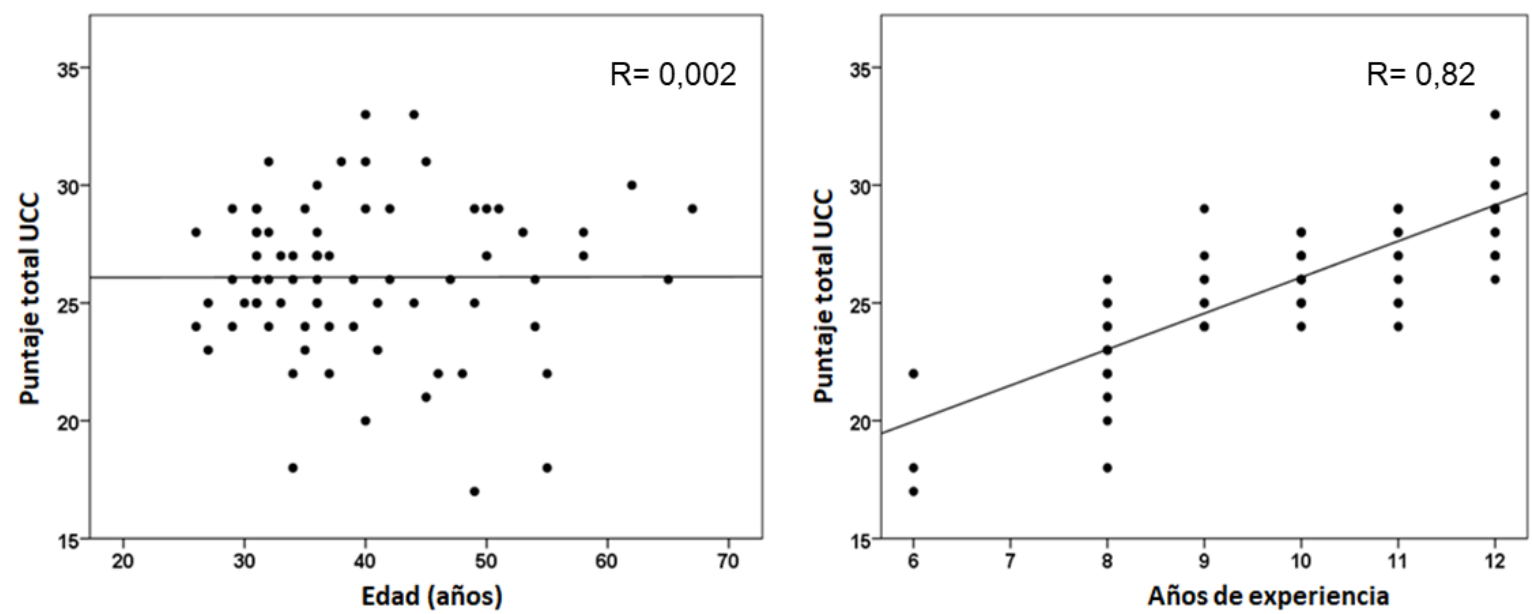

Figura 1. Relación entre la edad y años de experiencia docente con el uso del conocimiento científico.

\section{DISCUSIÓN}


El objetivo del estudio fue verificar las diferencias del uso del conocimiento científico por género, grado académico y experiencia docente y buscar sus posibles relacione entre variables. Los resultados han demostrado que no hubo diferencias en el UCC por género, grado académico y experiencia docente, no hubo relación entre la edad y el UCC, sin embargo, la experiencia docente se relacionó positivamente con el UCC.

Estos hallazgos son consistentes con otros estudios, puesto que los investigadores en la academia y el tipo de canal que utilizan para acceder a la información científica se suele reflejar en los años de experiencia profesional (Järvelin \& Ingwersen, 2004; Pontis \& Blandford, 2015).

La experiencia profesional, podría garantizar la capacidad de identificar los tipos de buscadores de información, ya que los expertos suelen distinguir más rápidamente la información relevante de la irrelevante (Vakkari, 2002). Por lo que los académicos con experiencia, interactúan con los dominios de la literatura científica para dar sentido a las situaciones problemáticas y resolver una amplia gama de actividades (Pontis \& Blandford, 2017), lo que posiblemente, en el caso de los menos experimentados, no podían resolver con amplia facilidad.

Esta información obtenida en este estudio en el que la experiencia es determinante en el UCC, se destaca que puede contribuir al proceso de alfabetización de los estudiantes de pregrado, puesto que el amplio repertorio científico puede contribuir en la formación de futuros científicos y diversos graduados universitarios (Miller et al., 2010). Además, la investigación ha indicado que los experimentados tienen un repertorio más diverso y robusto de estrategias que los novatos (Klein et al., 2007).

En consecuencia, los formuladores de políticas deben, por lo tanto, ser conscientes de la relación entre creación del valor científico, captura y las necesidades subyacentes de los científicos experimentados (Beck et al., 2019), puesto que esta amplia gama de habilidades y dominios pueden traer múltiples beneficios no sólo para las instituciones de educación superior, programas de posgrado, sino también para centros de investigación nacionales e internacionales.

También es lógico destacar que los jóvenes investigadores (noveles), podrían adquirir experiencia y habilidades de forma acelerada y mejor aún si es con la orientación de investigadores seniors. Una prueba de ello, es que los jóvenes investigadores son atraídos por programas de posgrado, comenzando en la fase doctoral, con el propósito de atraer a los mejores candidatos internacionales (LERU, 2018) para así garantizar un rápido crecimiento en la investigación científica y su respectivo incremento en número de trabajos de investigación y el aumentado de la visibilidad a nivel mundial.

De hecho, los resultados obtenidos en este estudio, son una clara muestra que los académicos de esta institución al parecer van por el camino del desarrollo científico, lo que futuramente se evidenciará en el incremento de investigaciones y el fortalecimiento de la alfabetización de los alumnos del pregrado. Por lo tanto, las experiencias de investigación pueden mejorar el desarrollo profesional y establecer los fundamentos para el aprendizaje y la toma de conciencia del UCC.

El estudio tiene algunas limitaciones que deben ser consideradas, dado que el tamaño de la muestra es relativamente pequeño y debido al tipo de selección de la muestra, los resultados podrían no pueden ser generalizados, aunque, sin desmerecer los resultados obtenidos, este es uno de los primeros que se han efectuado en la región del Maule, por lo 
que puede servir de línea de base para futuros estudios, inclusive para sensibilizar a los universidades e investigadores.

En conclusión, esta investigación demostró que la experiencia profesional es determinante en el UCC en una universidad regional, por lo que los resultados sugieren que las instituciones de educación superior deben vincular investigadores para fortalecer la alfabetización científica de los alumnos del pregrado.

\section{REFERENCIAS BIBLIOGRÁFICAS}

1. JÄRVELIN, K., \& INGWERSEN, P. (2004). Information seeking research needs extension towards tasks and technology. Information Research, 10(1), paper 212.

2. PONTIS, S., \& BLANDFORD, A. (2015). Understanding "influence": An exploratory study of academics' process of knowledge construction through iterative and interactive information seeking. Journal of the Association for Information Science and Technology, 68(8), 1576-1593.

3. PONTIS, S., \& BLANDFORD, A. (2015), Understanding "Influence:" An Exploratory Study of Academics' Process of Knowledge Construction Through Iterative and Interactive Information Seeking. J Assn Inf Sci Tec, 66: 1576-1593. doi:10.1002/asi.23277

4. VAKKARI, P. (2002). Subject knowledge, source of terms, and term selection in query expansion: An analytical study. In F. Crestani, M. Girolami, \& C. Joost van Rijsbergen (Eds.), Advances in information retrieval (pp. 110-123). Berlin, Germany: Springer Berlin Heidelberg

5. KLEIN, G., PHILliPS, J.K., RALL, E.L., \& PELUSO, D.A. (2007). A data-frame theory of sensemaking. In Expertise out of context Proceedings of the Sixth International Conference on Naturalistic Decision Making (pp. 113-155). Erlbaum

6. BECK, S., MAHDAD, M., BEUKEL, K., \& POETZ, M. (2019). The Value of Scientific Knowledge Dissemination for Scientists: A Value Capture Perspective. PUBLICATIONS, 7, 54; doi:10.3390/publications7030054

7. LERU position paper (2018). Delivering talent: Careers of researchers inside and outside academia. Thematic Group Research Careers, June 2018. https://www.leru.org/files/LERU-PP-DeliveringTalent 2018-June.pdf

8. COSSIO-BOLAÑOS, M., VIDAL ESPINOZA, R., YÁÑEZ SILVA, A., \& GÓMEZCAMPOS, R. (2014). Uso del conocimiento científico de estudiantes de pre-grado en una facultad de ciencias de la educación. Multiciencias, 14(3): 304-310.

9. SANUDO-GUERRA, MI., \& PERALES PONCE, R. (2014). Aprender ciencia para el bien común. Perfiles educativos, México, 36(143): 29-38.

10. ZEITONE, A. (2013). "Understanding the Nature of Scientific Enterprise in Light. of the Project (2061) Criteria and its Relationship to Some Demographic Variables". Jordanian journal in science education, 9(2), 119-139

11. SAMARA, S. (2015). Understanding of the 'nature of science' among undergraduate students at mutah university in jordan. European Scientific Journal March. Edition 11(8): 290-302 
12. MILlER, MC., MONTPLAISIR, LM., OFFERDAHL, EG., CHENG, FC., \& KETTERLING, GL. (2010). Comparison of views of the nature of science between natural science and nonscience majors. CBE Life Sci Educ. 9(1):45-54. doi:10.1187/cbe.09-05-0029

13. BECK, S., MAHDAD, M., BEUKEL, K., \& MARION, P. (2019). The Value of Scientific Knowledge Dissemination for Scientists - A Value Capture Perspective Publications 7, 54; doi:10.3390/publications7030054

14. JAMALI, H.R., \& NICHOLAS, D. (2008). Information-seeking behaviour of physicists and astronomers. Aslib Proceedings (Vol. 60, 5, pp. 444-462). Bingley, UK: Emerald Group Publishing. 